\title{
Patients Aged 50 Years and Older Have Greater Complication Rates After Anterior Cruciate Ligament Reconstruction: A Large Database Study
}

\author{
Madeleine A. Salesky, B.A., Jacob F. Oeding, B.S., Alan L. Zhang, M.D.,
} C. Benjamin Ma, M.D., Brian T. Feeley, M.D., and Drew A. Lansdown, M.D.

\begin{abstract}
Purpose: To assess the use of anterior cruciate ligament (ACL) reconstruction in older adults and to compare postoperative complication and revision surgery rates between patients older than and younger than 50 years old. Methods: Retrospective data were obtained using the PearlDiver database for patients who underwent arthroscopic ACL reconstruction from January 2010 to December 2017. Trends in the annual performance of ACL reconstruction were determined using nonparametric test of trends of ranks. Patients in each age group were matched based on sex and the Charlson Comorbidity Index. The incidence of postoperative complications within 90 days and subsequent knee surgery within 2 years of ACL reconstruction was collected. Postoperative complication rates were compared between matched age groups using the $\chi^{2}$ test. Results: A total of 20,993 patients aged 50 years and older and 154,817 patients younger than 50 years underwent ACL reconstruction between 2010 and 2017. The use of ACL reconstruction in patients aged 50 years or older decreased over time $(P=.044)$. Patients aged 50 years or older were more likely to experience at least 1 postoperative complication within 90 days compared to patients younger than 50 years $(2.5 \%$ vs $2.1 \%, P=.007)$. Older patients were 1.3 times as likely to experience deep vein thrombosis $(P=.002)$ and 1.8 times as likely to experience pulmonary embolism $(P<.001)$. Younger patients were more likely to undergo subsequent ACL reconstruction and experience knee pain, stiffness, and wound infection postoperatively $(P<.001, P<.001, P=.041) .1 .6 \%$ of patients aged 50 years or older underwent total knee arthroplasty within 2 years. Conclusions: ACL reconstruction in patients aged 50 years or older is associated with greater complication rates but lower rates of subsequent knee surgery relative to patients younger than 50 years of age. Younger patients were more prone to surgical complications whereas older patients experienced more medical complications. The increased incidence of VTE in this population suggests that thrombotic prophylaxis may be considered. Level of Evidence: Level III, retrospective comparative observational trial.
\end{abstract}

A nterior cruciate ligament (ACL) reconstruction is performed to restore knee function and stability for patients with instability after ACL injury. In young patients who participate in cutting and pivoting activities, arthroscopic ACL reconstruction is the gold standard treatment to restore knee stability and reduce the risk of subsequent meniscus and cartilage injury. ${ }^{1,2}$ The

From the School of Medicine (M.A.S.) and Department of Orthopaedic Surgery (J.F.O., A.L.Z., C.B.M., B.T.F., D.A.L.), University of California San Francisco, San Francisco, California, U.S.A.

The authors report the following potential conflicts of interest or sources of funding: B.M. reports board membership with Stryker, Conmed, and Tornier, grants from Aseculap, Samumed, Zimmer, and Conmed; and royalties from Conmed, outside the submitted work. A.L.Z. reports consultant for Stryker and Depuy Mitek, outside the submitted work. B.F. reports stocks in Bioniks and Kaliber.ai. D.A.L. reports grants from the Arthroscopy Association of North America (Arthroscopy Research Grant), American Orthopaedic Society for Sports Medicine (Young Investigator Award), Arthritis Foundation (research funding); patent pending for suture anchor device; Consultant for Vericel, Inc.; and educational support from Arthrex/Evolution Surgical and majority of ACL reconstruction cases in the United States are performed in patients aged 10 to 29 years. ${ }^{3,4}$ In older patients, however, the use of surgical management for ACL deficiency has been controversial in the past due to concerns about the possibility of infection, reoperation, and increased joint stiffness postoperatively. ${ }^{5}$ Based on these concerns, patients

Stryker, outside the submitted work. Full ICMJE author disclosure forms are available for this article online, as supplementary material.

Received February 24, 2021; accepted August 30, 2021

Address correspondence to Drew A. Lansdown, M.D., Department of Orthopaedic Surgery, University of California, San Francisco, 1500 Owens St., Suite 170, San Francisco, CA 94158.E-mail:Drew.lansdown@ucsf.edu

(C) 2021 THE AUTHORS. Published by Elsevier Inc. on behalf of the Arthroscopy Association of North America. This is an open access article under the CC BY-NC-ND license (http://creativecommons.org/licenses/by-nc-nd/4.0/). 2666-061X/21291

https://doi.org/10.1016/j.asmr.2021.08.010 
aged 50 years or older old were traditionally referred to physical therapy and encouraged to modify physical activity before ACL reconstruction was considered. Older patients are increasingly active and involved in high-risk pivoting sports such as skiing, leading to an aversion to accepting potential knee instability and prompting the desire to undergo reconstruction despite the possible risk of complications. ${ }^{6,7}$ Importantly, however, data concerning the risk of possible complications remain limited for this group of older patients considering ACL reconstruction.

Several studies have assessed the differences in clinical and functional outcomes after ACL reconstruction in older and younger adult populations. ${ }^{8-13} \mathrm{~A}$ recent meta-analysis found no significant difference in clinical or arthrometric outcome measures after ACL reconstruction between patients older 40 years old and younger than 40 years old. ${ }^{9}$ A systematic review by Costa et al. ${ }^{14}$ similarly found that patients older than and younger than 50 years old experience comparable improvements in patient satisfaction and knee stability, although older patients reported a reduced rate of returning to sport after surgery. As the age range of patients choosing to undergo reconstruction continues to broaden, there remains uncertainty concerning the incidence of postoperative complications among older patients. In addition, the magnitude of increase in the use of ACL reconstruction in the active aging population over recent years remains unclear.

The purpose of this study was to assess the use of ACL reconstruction in older adults and to compare postoperative complication and revision surgery rates between patients older than and younger than 50 years old. We hypothesized that the performance of ACL reconstruction in older adults is increasing over time, and that rates of complications, especially venous thromboembolism (VTE) and stiffness, are significantly greater in older adults.

\section{Methods}

\section{Data Collection}

Retrospective data for this study were obtained from a commercially available database: the Mariner 2020 subset from PearlDiver (www.pearldiverinc.com; PearlDiver, Inc., Colorado Springs, CO). Patients who underwent arthroscopic ACL reconstruction from January 2010 through December 2017 were identified using Current Procedural Terminology (CPT) code CPT-29888. Patients with less than 2 years of postoperative data in the database were excluded. Patients with the following related procedures were excluded according to the criteria used by Cancienne et al. ${ }^{15}$ : open primary repair of knee collateral ligament (CPT-27405), open primary repair of knee cruciate ligament (CPT-27407), open primary repair of knee collateral and cruciate ligament (CPT-27409), open autologous chondrocyte implantation, knee (CPT-27412), osteochondral allograft, knee, open (CPT-27415), osteochondral autograft, knee, open (CPT-27416), open reconstruction of dislocating patella (CPT-27420), open reconstruction of dislocating patella with extensor realignment (CPT-27422), open lateral retinacular release (CPT-27425), open extra-articular ligamentous reconstruction (CPT-27427), open intra-articular ligamentous reconstruction (CPT-27428), open intra- and extraarticular ligamentous reconstruction (CPT-27429), arthroscopic knee osteochondral autograft implantation (CPT-29866), arthroscopic knee osteochondral allograft implantation (CPT-29867), and arthroscopically aided posterior cruciate ligament repair/augmentation or reconstruction (CPT-29889). ${ }^{16}$ No other patients were excluded.

Patients who met inclusion and exclusion criteria were grouped according to their age at the time of surgery: 50 years and older or younger than 50 years. Patient demographic information including sex and age was collected. The annual number of ACL reconstruction surgeries across all age groups was collected.

To control for possible confounding variables in postoperative outcomes analysis, we matched patients 1:1 in each age group by sex and Charlson Comorbidity Index. Due to limitations of the database, we were unable to control for other variables like body mass index, specific medical comorbidities, or surgical details. Patients in these matched cohorts who experienced postoperative complications after ACL reconstruction were identified using the International Classification of Diseases (ICD), Ninth Revision, codes, ICD, Tenth Revision, codes, and CPT codes for each complication within 90 days of surgery (Table 1).

Subsequent knee surgeries including revision surgeries were analyzed separately from medical and surgical complications that occurred within 90 days of ACL reconstruction. Patients in the matched cohorts who underwent subsequent ACL reconstruction were identified using the code CPT-29888 after the initial surgery within 2 years. Patients who underwent other subsequent arthroscopic knee procedures within 2 years of initial surgery were identified according to the codes in Table 1. Patients who converted to total knee arthroplasty (TKA) were identified using the code CPT-27447 within 2 years after ACL reconstruction. Due to the lack of laterality modifiers available in the database for CPT codes, we were unable to account for laterality of the index surgery and subsequent procedures.

\section{Statistical Analysis}

Trends in the use of ACL reconstruction in each age group were determined using a nonparametric test of trends of ranks across ordered groups. The incidence of postoperative complications and subsequent surgery was compared between groups using the $\chi^{2}$ test. 
Table 1. Codes Used to Define Postoperative Complications and Subsequent Knee Surgeries

\begin{tabular}{ll}
\hline Postoperative Complications \\
\hline Knee pain
\end{tabular}

Knee stiffness

Pulmonary embolism Deep vein thrombosis

Pneumonia

Acute kidney injury

Infection or drainage

Disruption of wound

Subsequent knee surgeries

Subsequent knee surgery

Subsequent ACL reconstruction

Subsequent meniscus debridement or repair

Subsequent knee arthroscopy with lysis of adhesions, with or without manipulation

Subsequent manipulation of knee joint under general anesthesia (includes application of traction or other fixation devices)

Total knee arthroplasty
Codes

ICD-9-D-33828, ICD-9-D-33829, ICD-9-D-71946, ICD-9-D-71940, ICD-9-D-71948, ICD-10-D-M25561, ICD-10-D-M25562, ICD-10D-M25569, ICD-10-D-M79669

ICD-9-D-71846, ICD-9-D-71856, CPT-27570, ICD-10-D-M24561, ICD-10-D-M24562, ICD-10-D-M24569, ICD-10-P-9WB6XLZ, ICD-10-D-M25661, ICD-10-D-M25662, ICD-10-D-M25669 ICD-9-D-4151:ICD-9-D-4159, ICD-10-D-I26:ICD-10-D-I269 ICD-9-D-4532, ICD-9-D-4533, ICD-9-D-4534, ICD-9-D-45382, ICD9-D-45384, ICD-9-D-45385, ICD-9-D-45386, ICD-9-D-45340, ICD-9-D-45341, ICD-9-D-45342, ICD-10-D-I82401, ICD-10-DI82402, ICD-10-D-I82403, ICD-10-D-I82409, ICD-10-D-I82491, ICD-10-D-I82492, ICD-10-D-I82493, ICD-10-D-I82499, ICD-10D-I824Y1, ICD-10-D-I824Y2, ICD-10-D-I824Y3, ICD-10-DI824Y9, ICD-10-D-I824Z1, ICD-10-D-I824Z2, ICD-10-D-I824Z3, ICD-10-D-I824Z9

ICD-9-D-4800:ICD-9-D-4809, ICD-9-D-481ICD-9-D-4820-9-D-4821, ICD-9-D-48230, ICD-9-D-48231, ICD-9-D-48232, ICD-9-D-48239, ICD-9-D-48240, ICD-9-D-48241, ICD-9-D-48242, ICD-9-D-48249, ICD-9-D-48281, ICD-9-D-48282, ICD-9-D-48283, ICD-9-D-48284, ICD-9-D-48289, ICD-9-D-4829, ICD-9-D-4830, ICD-9-D-4831, ICD-9-D-4838, ICD-9-D-4841, ICD-9-D-4843-9-D-4845, ICD-9-D4846, ICD-9-D-4847, ICD-9-D-4848, ICD-9-D-485, ICD-9-D-486, ICD-10-D-J12:ICD-10-D-J189

ICD-9-D-5845, ICD-9-D-5846, ICD-9-D-5847, ICD-9-D-5848, ICD9-D-5849, ICD-10-D-N17:ICD-10-D-N179

ICD-9-D-71106, ICD-9-D-71146, ICD-9-D-71186, ICD-9-D-71196, ICD-9-D-99851, ICD-10-D-M01X61, ICD-10-D-M01X62, ICD-10D-M01X69, CPT-10180, CPT-20005, CPT-27310, CPT-29871

ICD-9-D-99830, ICD-9-D-99831, ICD-9-D-99832, ICD-9-D-99833, ICD-10-D-T8130XA, ICD-10-D-T8130XD, ICD-10-D-T8130XS, ICD-10-D-T8131XA, ICD-10-D-T8131XD", "ICD-10-D-T8131XS, ICD-10-D-T8132XA, ICD-10-D-T8132XD, ICD-10-D-T8132XS, ICD-10-D-T8133XA ICD-10-D-T8133XD, ICD-10-D-T8133XS

СРT-29888, СРT-27570, СРТ-29884, СРТ-29880, СРТ-29881, CPT-29882, СРT-29883

CPT-29888

CPT-29880, CPT-29881, CPT-29882, CPT-29883

CPT-29884

CPT-27570

CPT-27447

NOTE. Table 1 lists the codes used to identify patients who experienced postoperative complications within 90 days of anterior cruciate ligament reconstruction as well as patients who underwent subsequent ipsilateral or contralateral knee surgery within 2 years of ACL reconstruction.

ACL, anterior cruciate ligament; CPT, Current Procedural Terminology; ICD, International Classification of Diseases.

Statistical analysis was performed on the PearlDiver server and in Stata version 16.1 (StataCorp, College Station, TX). Significance was defined as $P<.05$.

\section{Results}

\section{Patient Demographics}

Overall, a total of 20,993 patients 50 years old and older and 154,817 patients younger 50 years old met inclusion and exclusion criteria and underwent ACL reconstruction during the study period (Fig 1). Across the entire cohort, $49 \%$ of patients were male, and the mean patient age was 32 years (first to third interquartile, 19-43 years). Among patients at least 50 years old, 9,406 (45\%) were male, and the mean patient age was 56 years (first to third interquartile, 52-58 years). The annual number of ACL reconstruction surgeries from 2010 to 2017 in patients aged 50 years or older versus patients younger 50 years old is summarized in Table 2, including all primary and revision ACL reconstructions. The annual incidence of ACL reconstruction in patients at least 50 years old showed a decreasing trend from 2010 to 2017 ( $P=.044$, Table 2 ).

After matching patients in each age group by sex and Charlson Comorbidity Index, we were left with cohorts of 20,946 patients in the 50 years and older age group 


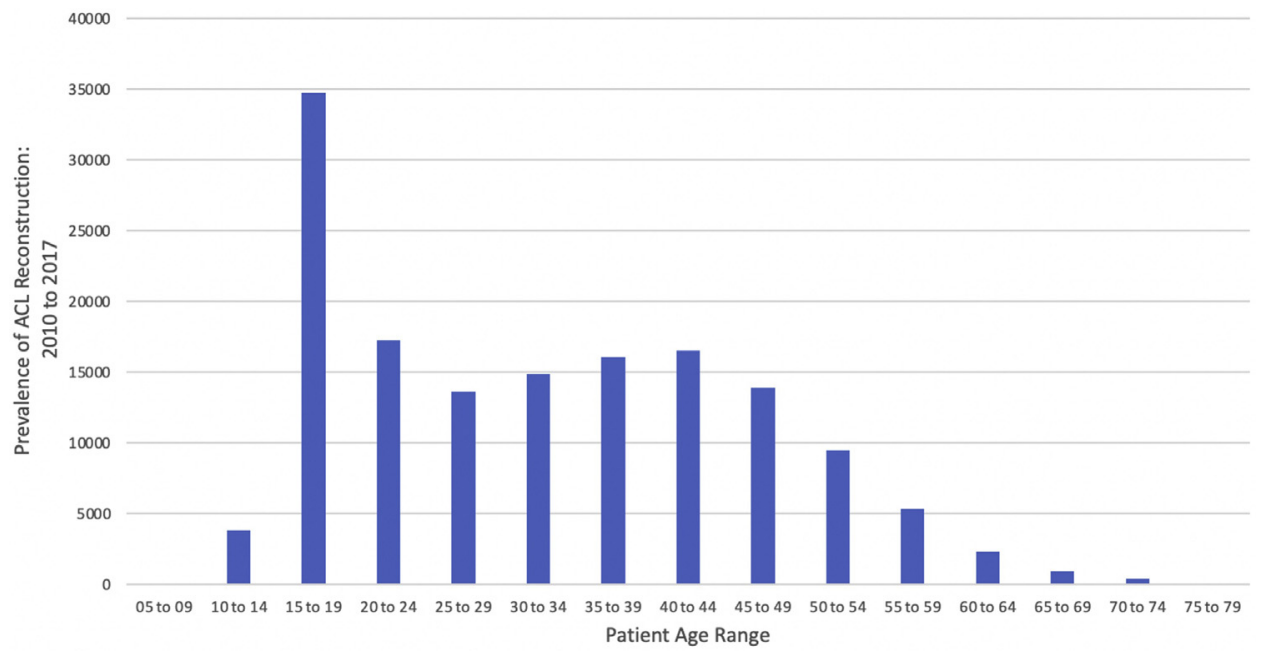

Fig 1. The distribution of patient age ranges is shown for all patients in the database who underwent anterior cruciate ligament (ACL) reconstruction from January 2010 to December 2017. The age range with the largest proportion of patients who underwent surgery was age 15 to 19 years. The majority of patients who underwent surgery during this time period were younger than 50 years old. and 20,946 patients in the younger than 50 years age group. In each age group, $45 \%$ of patients were male. Among matched patients at least 50 years old, the mean patient age was 56 years (first to third interquartile, $52-58$ years). Among matched patients younger than 50 years old, the mean patient age was 30 years (first to third interquartile, 18-40 years). These matched cohorts were used for analysis of postoperative complications and subsequent knee surgeries.

\section{Postoperative Complications}

Postoperative pain was the most common complication experienced in both cohorts, including $32 \%$ of patients 50 years and older and $35 \%$ of patients younger than 50 years. Excluding postoperative pain, $2.5 \%$ of patients aged 50 years old and older and $2.1 \%$ of patients younger than 50 years old experienced at least 1 other complication within 90 days of surgery, including pneumonia, VTE, acute kidney injury, wound disruption, infection, and stiffness $(P=.007$, Table 3$)$. Older patients were more likely to experience venous

Table 2. Number of ACL Reconstruction Surgeries by Year in Patients Older Than and Younger Than 50 Years of Age

\begin{tabular}{lcc}
\hline Year & Patients at Least 50 Years Old & $\begin{array}{c}\text { Patients Younger } \\
\text { than 50 Years Old }\end{array}$ \\
\hline Total $(\mathrm{N})$ & 20,993 & 154,817 \\
2010 & 3,005 & 22,115 \\
2011 & 2,784 & 21,578 \\
2012 & 2,658 & 21,502 \\
2013 & 2,729 & 21,633 \\
2014 & 2,863 & 21,679 \\
2015 & 2,661 & 20,989 \\
2016 & 2,488 & 18,800 \\
2017 & 2,149 & 15,600 \\
$P$ value & .044 & .038 \\
z-value & -2.02 & -2.08 \\
\hline
\end{tabular}

$\mathrm{ACL}$, anterior cruciate ligament. thromboembolic events (VTE), including pulmonary embolism (PE) and deep vein thrombosis (DVT), as well as acute kidney injury, within 90 days after ACL reconstruction (Table 4). Younger patients, however, were more likely to experience knee stiffness, knee pain, and wound infection after surgery (Table 4). The odds ratio (OR) of experiencing VTE (PE or DVT) (Table 4) was greater for patients 50 years old and older compared with patients younger than 50 years old (OR: $1.30,95 \%$ confidence interval [CI] 1.10-1.54) with the OR for DVT alone being 1.27 (95\% CI 1.07-1.52) and the OR for PE being 1.83 (95\% CI 1.28-2.62).

\section{Revision Knee Procedures and Conversion to TKA}

The rate of subsequent knee surgery within 2 years was significantly lower in patients 50 years old and older $(8.6 \%)$ relative to younger patients $(9.9 \%)$ (OR: 0.85 , Table 4). Patients 50 years old and older were less likely to undergo subsequent arthroscopic knee procedures (OR 0.72) and less likely to undergo subsequent ipsilateral or contralateral ACL reconstruction (OR 0.49) relative to younger patients (Table 4). The overall rate of TKA within 2 years of ACL reconstruction for patients 50 years old and older was $1.6 \%$.

\section{Discussion}

We observed that patients 50 years old and older were more likely to experience at least 1 postoperative complication within 90 days after ACL reconstruction relative to patients younger than 50 years old. Our initial hypothesis was partially confirmed because older patients had an increased risk of VTE, DVT, and PE after ACL reconstruction and increased risk of early conversion to TKA, although older patients were less likely to have subsequent knee surgery or experience knee pain, stiffness, or infection after surgery. There was also a decrease in the performance of ACL reconstruction in 
Table 3. Proportion of Patients with Any Postoperative Complication* Within 90 Days

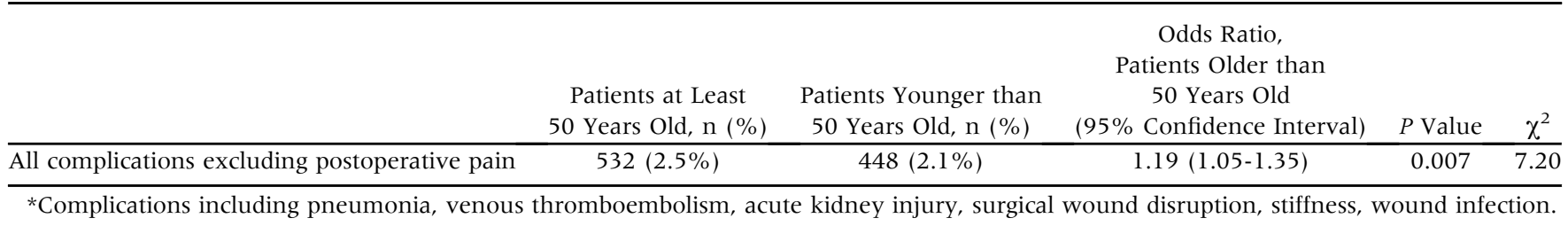

patients 50 years old and over from 2010 to $2017(P=$ .044), contrary to our initial hypothesis. The findings of this study can allow for informed discussions between surgeons and patients older than 50 years regarding the potential for risk after ACL reconstruction, especially as medical risks are observed more frequently in this group of patients.

Nonsurgical management of ACL injuries in older athletes has been advocated in the past due to concerns that ACL reconstruction in older patients would lead to increased knee stiffness, arthrofibrosis, infections, poor wound healing, and thromboembolic disease compared with younger patients. ${ }^{17}$ This has been refuted by studies reporting similar outcomes among younger and older patients after ACL reconstruction. ${ }^{1-13}$ Patientreported outcomes after nonoperative management of ACL rupture in older adults are also mixed. Ciccotti et al. ${ }^{18}$ reported an $83 \%$ satisfaction rate among patients 40 to 60 years old treated with nonoperative management, but $37 \%$ of patients experienced reinjury and all patients were required to modify their activities and lifestyle. Gföller et al. ${ }^{19}$ similarly found that subjective patient satisfaction improved over time after nonoperative management, but radiologic outcomes scores stayed constant or deteriorated and knee laxity testing showed increased instability over time. Physical activity levels also decreased significantly compared with preinjury levels. ${ }^{19}$ Given that nonoperative treatment is associated with chronic degenerative changes, progressive instability, and high risk of reinjury, this approach may be unacceptable for many older patients with ACL deficiency who desire to return to previous activity levels.

The overall complication rate of ACL reconstruction surgery was significantly greater in older patients relative to younger patients in this cohort. Importantly, multiple previous reports have described the clinical effectiveness of ACL reconstruction in these older patients. Barber et al. ${ }^{20}$ found no difference in patient satisfaction between patients older than 40 years (average 44 years) and younger than 40 (average 27 years), with $89 \%$ and $91 \%$ patient satisfaction respectively. A recent meta-analysis including four retrospective cohort studies and a total of 287 patients (129 older than 50 years, 158 younger than 50 years) found no difference in the improvement of International Knee Documentation Committee scores or Lysholm scores between patients older than and younger than 50 years old. $^{21}$ There was also no difference in Tegner activity scores or the incidence of knee AP laxity as measured on KT-1000 or KT-2000 arthrometer at final followup. ${ }^{21}$ All studies included in the meta-analysis reported

Table 4. Incidence of Postoperative Complications and Subsequent Knee Surgeries by Age Group

\begin{tabular}{|c|c|c|c|c|}
\hline & Patients $\geq 50$ Years & Patients $<50$ Years & $P$ Value & Odds Ratio $(95 \% \mathrm{CI})^{*}$ \\
\hline \multicolumn{5}{|l|}{ Postoperative complications } \\
\hline Total & 20,946 & 20,946 & & \\
\hline Knee pain & $6,683(31.9 \%)$ & $7,334(35.0 \%)$ & $<.001$ & $0.87(0.84-0.91)$ \\
\hline Knee stiffness & $819(3.9 \%)$ & $1,027(4.9 \%)$ & $<.001$ & $0.79(0.72-0.87)$ \\
\hline Venous thromboembolism (PE and DVT) & $327(1.6 \%)$ & $252(1.2 \%)$ & .002 & $1.30(1.10-1.54)$ \\
\hline DVT & $287(1.4 \%)$ & $226(1.1 \%)$ & .008 & $1.27(1.07-1.52)$ \\
\hline $\mathrm{PE}$ & $86(0.4 \%)$ & $47(0.2 \%)$ & $<.001$ & $1.83(1.28-2.62)$ \\
\hline Acute kidney injury & $56(0.3 \%)$ & $27(0.1 \%)$ & .002 & $2.08(1.31-3.29)$ \\
\hline Infection or drainage of surgical wound & $86(0.4 \%)$ & $116(0.6 \%)$ & .041 & $0.74(0.56-0.98)$ \\
\hline Disruption of wound & $40(0.2 \%)$ & $58(0.3 \%)$ & .086 & $0.69(0.46-1.03)$ \\
\hline Pneumonia & $93(0.4 \%)$ & $70(0.3 \%)$ & .084 & $1.33(0.97-1.81)$ \\
\hline \multicolumn{5}{|l|}{ Subsequent knee surgeries } \\
\hline Subsequent arthroscopic knee procedure & $1,534(7.3 \%)$ & $2,060(9.8 \%)$ & $<.001$ & $0.72(0.68-0.78)$ \\
\hline Subsequent ipsilateral or contralateral ACL reconstruction & $535(2.6 \%)$ & $1,058(5.1 \%)$ & $<.001$ & $0.49(0.44-0.55)$ \\
\hline Conversion to total knee arthroplasty & $345(1.6 \%)$ & $48(0.2 \%)$ & $<.001$ & $7.29(5.39-9.87)$ \\
\hline Any subsequent surgery (arthroscopic or total knee arthroplasty) & $1,802(8.6 \%)$ & $2,082(9.9 \%)$ & $<.001$ & $0.85(0.80-0.91)$ \\
\hline
\end{tabular}

ACL, anterior cruciate ligament; DVT, deep vein thrombosis; PE, pulmonary embolism.

*Odds ratio for patients 50 years old and older relative to patients younger than 50 years old. 
significant improvement in clinical outcomes across both patient age groups. ${ }^{21}$ These findings indicate that ACL reconstruction in patients older than 50 years old results in similar knee stability and function scores compared to younger patients. However, this prior meta-analysis was unable to assess for differences in complication rates due to their low incidence after ACL reconstruction and the limited number of patients included in the analysis. Compared with the study by Tan et al., ${ }^{21}$ the current study highlights this difference in complication rates, with the most notable difference being the rate of DVT/PE in older patients.

In our large cohort, we observed that patients 50 years old and older have a greater risk of DVT and PE compared with younger patients. These findings build off of those by Gaskill et al., ${ }^{22}$ who found that patients older than 35 years had increased odds of VTE after ACL reconstruction. VTE is a rare but serious complication after ACL reconstruction, with a recent systematic review reporting incidences of asymptomatic DVT, symptomatic DVT, and PE of $8.4 \%, 2.3 \%$, and $0.2 \%$, respectively. ${ }^{23}$ While we were unable to determine whether VTE was symptomatic or asymptomatic due to database limitations, the increased risk of thromboembolic disease in patients 50 years old and older reported in this study suggests that anticoagulation prophylaxis may be considered for older patients undergoing ACL reconstruction. Currently, there is no standardized recommendation for the use of VTE prophylaxis during ACL reconstruction. A recent survey study found that $50.7 \%$ of orthopedic surgeons routinely use chemical VTE prophylaxis, with $95 \%$ reporting using aspirin although no standardized dosing protocol was identified. ${ }^{24}$ Given the rate of DVT/PE, surgeons may consider thromboembolic prophylaxis following ACL reconstruction for older patients. Future studies should assess for the prevalence of thrombotic prophylaxis for knee arthroscopy and the association between prophylaxis and clinically significant VTE in this population.

We also observed that the incidence of knee stiffness was actually lower for patients 50 years old and older relative to younger patients in our cohort. The incidence of knee stiffness overall was also lower in our cohort $(4.4 \%)$ than in previous studies. Robertson et al. ${ }^{25}$ reported an incidence of knee stiffness of $12 \%$ at 6 months and $5 \%$ at 12 months postreconstruction, with incomplete physiotherapy and previous knee surgery being the strongest predictors of knee stiffness. There was no difference in age $(P=.70)$ between patients who experienced stiffness and those who did not. ${ }^{25}$ The decreased rate of knee stiffness in older patients cannot be fully explained with the data available, although could be due to graft choice, rehabilitation protocols, timing of surgery and administrative coding limitations, among other factors. Importantly, in this large cohort it does not appear that older patients have an increased risk of knee stiffness after ACL reconstruction.

Concerning the rate of subsequent knee surgery, we observed that younger patients were more likely to undergo additional knee arthroscopy including ipsilateral or contralateral ACL reconstruction within 2 years of the index ACL reconstruction. Part of this difference may be due to younger patients being more likely to undergo meniscal repair than meniscectomy, although we are unable to determine this conclusively given the study design and limitations. Contrastingly, patients 50 years old and older were more likely to undergo TKA within two years after ACL reconstruction compared to patients younger than 50 years old. These findings align with prior literature which identified older patient age at ACL reconstruction as a predictor of developing knee osteoarthritis. ${ }^{26}$ Overall, however, older patients were less likely to undergo subsequent surgery including arthroscopic surgery or TKA within 2 years compared with younger patients. Future studies should assess the mechanism by which ACL reconstruction influences TKA rates among older patients. The increased risk of TKA for older patients after ACL reconstruction compared with patients without ACL reconstruction can inform preoperative planning and assist surgeons in counseling patients about potential treatments.

\section{Limitations}

There are limitations to this study. Data analysis was limited to variables that were available in the database, as we did not have direct access to patient charts. Patient information including race and ethnicity, income, symptom severity, and duration of symptoms was unavailable. Patient-reported outcome measures and imaging reports are also not included in the database. Additionally, we were unable to assess the time from injury to ACL repair or cartilage status at the time of ACL repair. Notably, because laterality modifiers were not readily available for CPT codes, the laterality of primary and subsequent knee surgeries cannot be determined. Therefore, it is not clear whether the reported subsequent knee surgeries were a primary procedure on the contralateral leg, or a primary or revision procedure on the ipsilateral leg. Our study spans the introduction of ICD-10 codes, so the recording of postoperative complications was dependent upon the coding system used in a given year. Inconsistencies in how postoperative complications were recorded may have influenced our findings, as is the case with all retrospective and observational database studies. The use of autograft or allograft tissue, as well as primary or revision reconstruction surgery, cannot be discerned from CPT codes alone, so no recommendations can be drawn regarding graft type or approach to revision surgeries with these data. Despite these limitations, we believe that the findings from this large cohort of 
patients provides valuable insight into the incidence of postoperative complications in older patients after ACL reconstruction. Future studies can build off of these findings to explore the impact of additional variables on postoperative outcomes for older and younger patients after ACL reconstruction.

\section{Conclusions}

ACL reconstruction in patients aged 50 years or older is associated with greater complication rates but lower rates of subsequent knee surgery relative to patients younger than 50 years of age. Younger patients were more prone to surgical complications while older patients experienced more medical complications. The increased incidence of VTE in this population suggests that thrombotic prophylaxis may be considered.

\section{References}

1. Gabler CM, Jacobs CA, Howard JS, Mattacola CG, Johnson DL. Comparison of graft failure rate between autografts placed via an anatomic anterior cruciate ligament reconstruction technique: A systematic review, meta-analysis, and meta-regression. Am J Sports Med 2016;44:1069-2079.

2. Filbay SR, Grindem H. Evidence-based recommendations for the management of anterior cruciate ligament (ACL) rupture. Best Pract Res Clin Rheumatol 2019;33:33-47.

3. Leathers MP, Merz A, Wong J, Scott T, Wang JC, Hame SL. Trends and demographics in anterior cruciate ligament reconstruction in the United States. J Knee Surg 2015;28:390-394.

4. Herzog MM, Marshall SW, Lund JL, Pate V, Mack CD, Spang JT. Trends in incidence of ACL reconstruction and concomitant procedures among commercially insured individuals in the United States, 2002-2014. Sports Health 2018;10:523-531.

5. Buss DD, Min R, Skyhar M, Galinat B, Warren RF, Wickiewicz TL. Nonoperative treatment of acute anterior cruciate ligament injuries in a selected group of patients. Am J Sports Med 1995;23:160-165.

6. Seng K, Appleby D, Lubowitz JH. Operative versus nonoperative treatment of anterior cruciate ligament rupture in patients aged 40 years or older: An expectedvalue decision analysis. Arthroscopy 2008;24:914-920.

7. Desai N, Björnsson H, Samuelsson K, Karlsson J, Forssblad M. Outcomes after ACL reconstruction with focus on older patients: Results from The Swedish National Anterior Cruciate Ligament Register. Knee Surg Sports Traumatol Arthrosc 2014;22:379-386.

8. Wierer G, Runer A, Hoser C, Herbst E, Gföller P, Fink C. Acute ACL reconstruction in patients over 40 years of age. Knee Surg Sports Traumatol Arthrosc 2017;25:1528-1534.

9. Kim KT, Kim HJ, Lee HI, et al. A comparison of results after anterior cruciate ligament reconstruction in over 40 and under 40 years of age: A meta-analysis. Knee Surg Relat Res 2018;30:95-106.
10. Brandsson S, Kartus J, Larsson J, Eriksson BI, Karlsson J. A comparison of results in middle-aged and young patients after anterior cruciate ligament reconstruction. Arthroscopy 2000;16:178-182.

11. Best MJ, Zikria BA, Wilckens JH. Anterior cruciate ligament injuries in the older athlete. Sports Health 2021;13:285-289.

12. Brown CA, McAdams TR, Harris AH, Maffulli N, Safran MR. ACL reconstruction in patients aged 40 years and older: A systematic review and introduction of a new methodology score for ACL studies. Am J Sports Med 2013;41:2181-2190.

13. Mall NA, Frank RM, Saltzman BM, Cole BJ, Bach BR Jr. Results after anterior cruciate ligament reconstruction in patients older than 40 years: How do they compare with younger patients? A systematic review and comparison with younger populations. Sports Health 2016;8:177-181.

14. Costa GG, Grassi A, Perelli S, et al. Age over 50 years is not a contraindication for anterior cruciate ligament reconstruction. Knee Surg Sports Traumatol Arthrosc 2019;27:3679-3691.

15. Cancienne JM, Gwathmey FW, Miller MD, Werner BC. Tobacco use is associated with increased complications after anterior cruciate ligament reconstruction. Am J Sports Med 2016;44:99-104.

16. Cancienne JM, Brockmeier SF, Gulotta LV, Dines DM, Werner BC. Ambulatory total shoulder arthroplasty: A comprehensive analysis of current trends, complications, readmissions, and costs. J Bone Joint Surg Am 2017;99: 629-637.

17. Legnani C, Terzaghi C, Borgo E, Ventura A. Management of anterior cruciate ligament rupture in patients aged 40 years and older. J Orthop Traumatol 201 1;12:177-184.

18. Ciccotti MG, Lombardo SJ, Nonweiler B, Pink M. Nonoperative treatment of ruptures of the anterior cruciate ligament in middle-aged patients. Results after long-term follow-up. J Bone Joint Surg Am 1994;76:1315-1321.

19. Gföller P, Abermann E, Runer A, et al. Non-operative treatment of ACL injury is associated with opposing subjective and objective outcomes over 20 years of follow-up. Knee Surg Sports Traumatol Arthrosc 2019;27:2665-2671.

20. Barber FA, Elrod BF, McGuire DA, Paulos LE. Is an anterior cruciate ligament reconstruction outcome age dependent? Arthroscopy 1996;12:720-725.

21. Tan CW, Hsu WH, Yu PA, et al. Anterior cruciate ligament reconstruction in patients older than 50 years: A systematic review and meta-analysis. Orthop J Sports Med 2020;8: 2325967120915698.

22. Gaskill T, Pullen M, Bryant B, Sicignano N, Evans AM, DeMaio M. The prevalence of symptomatic deep venous thrombosis and pulmonary embolism after anterior cruciate ligament reconstruction. Am J Sports Med 2015;43: 2714-2719.

23. Erickson BJ, Saltzman BM, Campbell KA, Fillingham YA, et al. Rates of deep venous thrombosis and pulmonary embolus after anterior cruciate ligament reconstruction: A systematic review. Sports Health 2015;7:261-266.

24. Keller RA, Moutzouros V, Dines JS, Bush-Joseph CA, Limpisvasti O. Deep venous thrombosis prophylaxis in anterior cruciate ligament reconstructive surgery: What is the current state of practice? Sports Health 2018;10:156-159. 
25. Robertson GA, Coleman SG, Keating JF. Knee stiffness following anterior cruciate ligament reconstruction: The incidence and associated factors of knee stiffness following anterior cruciate ligament reconstruction. Knee 2009;16: 245-247.
26. Shelbourne KD, Benner RW, Gray T. Results of anterior cruciate ligament reconstruction with patellar tendon autografts: Objective factors associated with the development of osteoarthritis at 20 to 33 years after surgery. Am J Sports Med 2017;45:2730-2738. 